\title{
Influence of Functionalized Multiwall Carbon Nano-Tube on the Structure and Performance of Cellulose Di-Acetate Based Forward Osmotic Membrane for Desalination Process
}

\author{
Xiulan Zhao ${ }^{1,2}$, Jianfang Sheng ${ }^{1}$, Lijing Zhu ${ }^{1}$, Anjiang Zhang ${ }^{1 *}$ and Lixin Xue (1,2* $^{\text {. }}$
}

${ }^{1}$ Polymer and Composite Division, Key Laboratory of Marine Materials and Related Technologies, Zhejiang Key Laboratory of Marine Materials and Protective Technologies, Ningbo Institute of Materials Technology and Engineering, Chinese Academy of Sciences, Ningbo 315201, P.R. China

${ }^{2}$ Ningbo Lianhua Environmental Science and Technology Co., Ltd, Ningbo 315400, P. R. China

\begin{abstract}
To improve the performance of Cellulose di-acetate (CDA) based forward osmotic (FO) membrane in sea water desalination process, functionalized multi-walled carbon nano-tubes (MWCNTs) were blended as additives at varied compositions, from 0 to $5 \mathrm{wt} \%$, into the solutions to prepare FO membranes using a classical phase-inversion method. The structure and property of the formed membranes were characterized by Fourier transfer infrared (FTIR) spectroscopy, Atomic Force Microscopy (AFM), Scanning Electron Microscopy (SEM), Transitional Electron Microscopy (TEM), Water Flux and Reverse Solute Flux Tests. It was found that the content of functionalized MWCNTs was an important factor influencing the morphology, porous structures and properties of the blend membranes. SEM, AFM and TEM images of the blend membranes showed that the surface morphology and the cross-sectional morphology changed with the content of functionalized MWCNTs. It is interesting to see that at the presence of functionalized MWCNTs, the surface contact angle and the reverse solute flux of the FO membranes could be greatly improved without significantly affecting the pure water flux. With the addition of only about 1 wt $\%$ MWCNTs, the water flux of CDA based FO membrane was increased from 10.5 to $12.5 \mathrm{~L} / \mathrm{m}^{2} \mathrm{~h}$ while its reverse solute flux was reduced from 1.8 to below $0.3 \mathrm{~mol} / \mathrm{m}^{2} \mathrm{~h}$. Desalination tests with $3.5 \mathrm{wt} \%$ simulated seawater feed solution had shown that the blend membrane with $1 \mathrm{wt} \%$ MWCNTs, was $366 \%$ higher in water flux and $53 \%$ lower in reverse solute flux than those of pure CDA FO membrane. These results suggest that CDA bases FO membranes modified with functionalized MWCNTs could possess good potential to be further developed for practical applications in the sea water desalination processes.
\end{abstract}

Keywords: Forward osmosis; Multi-walled carbon nanotubes; CDA; Blend membrane

\section{Introduction}

In recent years, Forward Osmosis (FO), as an emerging water treatment technology, has gained increasing interest. Compared to Reverse Osmosis (RO), FO has many potential advantages: (1) it has high rejection for a wide range of contaminants, and less membrane fouling [1-4]; (2) it is a spontaneous low pressure process that needs lower external energy. As a low pressure membrane separation process, it is expected to lead to energy-efficient separations in many areas including seawater desalination [5,6], food processing [7-9], waste water treatment [10-12], electric power production [13-15], pharmaceutical industry $[16,17]$ and so on. Even though a lot have been achieved relating to FO technology $[18,19]$, the development of high water flux FO membrane with optimized structures remain as the central task for increasing the efficiency of the processes. Elimelech et al. concluded that neither cellulose triacetate RO membrane, nor polyamide RO composite membrane could be used for FO processes due to low water flux of $1-2 \mathrm{~L} / \mathrm{m}^{2} . \mathrm{h}$ [5]. They showed that commercialized cellulose triacetate (CTA) FO membranes with optimized structure had higher water flux of $6-8 \mathrm{~L} / \mathrm{m}^{2} \cdot \mathrm{h}$, but these water flux values were still too low for practical applications.

Recently, researchers are interested in the potential of using Multi-walled carbon nano-tubes (MWCNTs), as fillers to improve the properties of nano-composite membranes [20,21]. It was hypothesized that MWCNTs could increase water permeation by disrupting polymer chain packing to form external and internal nanoscale channels [22,23]. A high loading of MWCNTs tend to form a porous network for efficient water transport while the mechanical property and contaminant rejections of the membranes were both improved at the same time $[22,24]$. Shawky's results suggested that the addition of MWCNTs improved the rejection of both salt and organic matter for PA base FO membrane [25]. Zhang et al. introduced MWCNTs functionalized with isocyanate and 5-isocyanato-isophthaloyl chloride into PSF ultrafiltration membranes, and found that MWCNTs influenced surface mean pore size and porosity of membrane. As a result, with an appropriate amount of modified MWCNTs, the water flux of the membrane was enhanced [20].

In this paper, to investigate the effect of MWCNTs on the structure and properties of FO membranes, MWCNTs were incorporated into the CDA matrix during the phase inversion process. The concentration of nano-tubes in the polymer matrix was controlled from 0 to $5 \mathrm{wt} \%$

*Corresponding authors: Lixin Xue, Ningbo Institute of Materials Technology and Engineering, Chinese Academy if Sciences, E-mail: xuelx@nimte.ac.cn

Anjiang Zhang, Ningbo Institute of Materials Technology and Engineering, Chinese Academy of Sciences, No. 1219, Zhongguan West Road, Zhenhai District, Ningbo, Zhejiang Province, P. R. China 315201, E-mail: zhanganjiang@nimte.ac.cn

Received July 31, 2015; Accepted February 28, 2015; Published August 30 2015

Citation: Zhao X, Sheng J, Zhu L, Zhang A, Xue L (2015) Influence of Functionalized Multiwall Carbon Nano-Tube on the Structure and Performance of Cellulose Di-Acetate Based Forward Osmotic Membrane for Desalination Process. J Membra Sci Technol 5: 135. doi:10.4172/2155-9589.1000135

Copyright: @ 2015 Zhao X, et al. This is an open-access article distributed under the terms of the Creative Commons Attribution License, which permits unrestricted use, distribution, and reproduction in any medium, provided the original author and source are credited. 
Citation: Zhao X, Sheng J, Zhu L, Zhang A, Xue L (2015) Influence of Functionalized Multiwall Carbon Nano-Tube on the Structure and Performance of Cellulose Di-Acetate Based Forward Osmotic Membrane for Desalination Process. J Membra Sci Technol 5: 135. doi:10.4172/21559589.1000135

Page 2 of 6

MWCNTs were evaluated by TEM and Fourier transform infrared (FT-IR). The physicochemical properties of the mixed matrix membranes were evaluated by scanning electron microscopy (SEM), FT-IR spectroscopy, Atomic Force Microscope (AFM) and contact angle measurements. The membrane performances were assessed by water flux and the reverse solute flux.

\section{Experimental}

\section{Materials}

MWCNTs were obtained from Chengdu organic chemicals co., LTD. Chinese Academy of Sciences (Chengdu, China).Cellulose Diacetate (CDA) with an acylation degree of $39.2 \%$ was purchased from Aladdin Reagent Co., Ltd. Analytical grade sodium chloride, acetone, Methanol, Lactic acid, 1,4-dioxane were obtained from Sinopharm. De-ionized water was used for the water flux and the reverse solute flux.

\section{Membrane Preparation}

To fabricate blend membrane, the casting solution was formed by dissolving CDA and MWCNTs in 1, 4-dioxane, acetone with Methanol and Lactic acid additives under stirring at $50^{\circ} \mathrm{C}$. The concentration of MWCNTs ranged in 1, 2, 3, 4 and $5 \mathrm{wt} \%$ relative to the weight of CDA, respectively. After being fully dissolved, the casting solution was put still to remove gas and then spread onto a polyester mesh with a casting $\mathrm{knife}$ of $150 \mu \mathrm{m}$. The above membranes were left in the air for about $30 \mathrm{~s}$ and then immersed into a water bath. Then the nascent membrane was annealed in hot water at $85^{\circ} \mathrm{C}$ for $10 \mathrm{~min}$ and then stored in de-ionized water prior to use. The obtained membranes were named as M-1, M-2, M-3, M-4 and M-5, respectively. The pure CDA membrane without MWCNTs loading was also prepared and named as M0.

\section{Characterization of membrane}

The chemical compositions of the neat MWCNTs, M-0 and M-5 were measured using a Thermo-Nicolet 6700 FT-IR spectrometer (US) over a range of $4000-400 \mathrm{~cm}^{-1}$. The cross morphologies of the membranes were characterized using a Hitachi S\#4800 SEM (Japan) system. All samples were sputtered gold for 2 minutes before observation.

The microstructures of MWCNTs were characterized using a Tecnai G2 F20 TEM at an accelerating voltage of $100 \mathrm{kV}$. MWCNTs were dispersed in methylbenzene by ultrasound and some pieces were collected on carbon-coated 300-mesh copper grids for observation.

The morphologies of membranes were observed by Keyence VKX200K scanning microscope (Japan). The samples were operated at room temperature.

AFM images of the membrane surfaces were taken on a Dimension 3100V SPM system from Veeco (US) with tapping mode. The samples were operated at room temperature with the scanning size of $5 \mu \mathrm{m} \times 5 \mu \mathrm{m}$.

The hydrophilicity of the membranes was characterized by water contact angle measurements (CA, OCA20, Data physics, Germany). A piece of $1 \times 5 \mathrm{~cm}^{2}$ membrane sample was attached onto a glass slide. $1.0 \mu \mathrm{L}$ of distilled water was dropped onto the air-side surface of the membrane at room temperature. The water contact angle decaying with drop age was recorded using speed optimum video measurement technology. At least ten measurements were averaged to get a reliable value.

\section{Testing of Membrane Filtration Performance in Forward Osmosis Mode}

The filtration performance of the membrane was measured by a cross flow filtration system [26-28] with an effective membrane area of $64.26 \mathrm{~cm}^{2}$. The volume of both feed and draw solutions was $4.0 \mathrm{~L}$ at the start of each experimental run, a $2.0 \mathrm{M} \mathrm{NaCl}$ solution was used as draw solution and the de-ionized water was used as feed solution. The feed and draw solution was circulated at a rate of $0.5 \mathrm{~L} / \mathrm{min}$ in a closed loop driven by gear pumps (Figure 1). A balance (SE6001F, USA) connected to the computer recorded down the mass of water permeating into draw solution while a conductivity meter was used to record down the concentration of $\mathrm{NaCl}$ in feed solution. The water flux $\left(\mathrm{Jw}, \mathrm{L} / \mathrm{m}^{2} \mathrm{~h}\right)$ was calculated from the weight changes draw solution [26].

$$
J_{W}=\frac{\Delta V}{S_{m} \Delta t}
$$

Where $\Delta \mathrm{V}$ is the change in volume of water collected over a predetermined time, $\Delta \mathrm{t}$ is the FO process duration; $\mathrm{S}_{\mathrm{m}}$ is the effective membrane surface area $\left(\mathrm{m}^{2}\right)$.

The salt concentration in the feed water was determined from the conductivity measurement using a calibration curve for the single salt solution. And the reverse solute flux was calculated by the change of $\mathrm{NaCl}$ concentration in the feed solution. The reverse solute flux $\mathrm{J}_{\mathrm{s}}(\mathrm{mol} /$ $\mathrm{m}^{2} \mathrm{~h}$ ) is determined from the increase of the feed conductivity [26]:

$$
J_{s}=\frac{\Delta\left(C_{t} V_{t}\right)}{S_{m} \Delta t}
$$

Where $\mathrm{C}_{t}$ and $\mathrm{V}_{t}$ are the salt concentration and the volume of the feed at the end of FO tests, respectively. At least five samples were tested and the average value was obtained for each membrane.

\section{Texting on osmotic seawater desalination}

The membranes were tested under forward osmosis (FO) mode where the feed solution side faces against the dense selective layer. The volume of both feed and draw solutions was $2.0 \mathrm{~L}$ at the start of each experimental run, a $2.0 \mathrm{M} \mathrm{NaCl}$ solution was used as draw solution and the seawater (3.5 wt\%) was used as feed solution. The feed and draw solution was circulated at a rate of $0.5 \mathrm{~L} / \mathrm{min}$ in a closed loop driven by gear pumps. At least five samples were tested and the average value was obtained for each membrane.

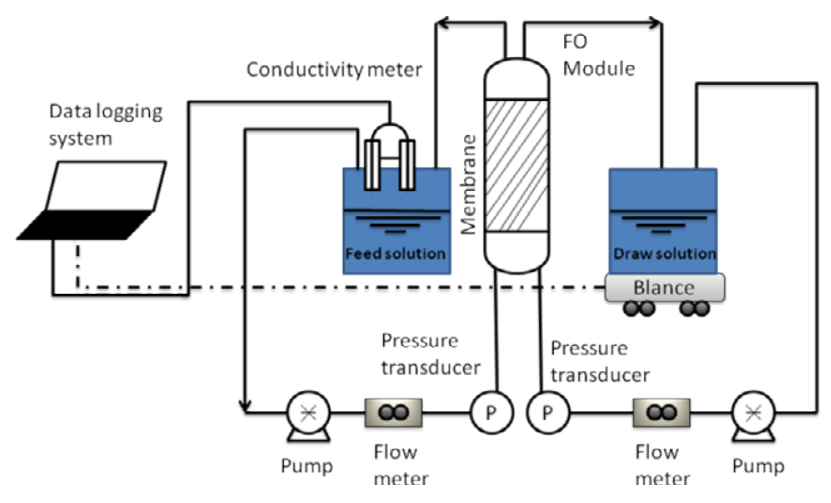

Figure 1: Schematic diagram of FO system [26] 
Citation: Zhao X, Sheng J, Zhu L, Zhang A, Xue L (2015) Influence of Functionalized Multiwall Carbon Nano-Tube on the Structure and Performance of Cellulose Di-Acetate Based Forward Osmotic Membrane for Desalination Process. J Membra Sci Technol 5: 135. doi:10.4172/21559589.1000135

\section{Results and Discussion}

\section{The microstructures and FT-IR spectra of MWCNTs and FT- IR spectra of the blend membrane}

MWCNTs were evaluated by TEM and FT-IR. Figure 2 shows TEM images of the functionalized MWCNTs. The TEM images show the average diameter of MWCNTs is about $20 \mathrm{~nm}$. Figure 3 shows the FTIR spectra of the functionalized MWCNTs, pure CDA membrane (M-0) and a typical blend membrane (M-5). For MWCNTs, the peak at $1578 \mathrm{~cm}^{-1}$ is attributed to the vibration of carbon skeleton of the nanotubes [29]. Additional peaks appeared at around 3430, 1720 and $1213 \mathrm{~cm}^{-1}$ are assigned to $-\mathrm{OH}, \mathrm{C}=\mathrm{O}$, and $\mathrm{C}-\mathrm{C}-\mathrm{O}$ stretching present in carboxylic groups $(-\mathrm{COOH})$, respectively [30]. The peaks suggested the MWCNTs were successfully oxidized. The FT-IR spectra of the pure and blend membranes are presented in Figure 3. The spectra of blend membranes are not noticeably different from that of the pure CDA membrane. However, a new tiny peak at $1324 \mathrm{~cm}^{-1}$ still can be found, corresponding to the $-\mathrm{OH}$ vibration of carboxyl groups by the added MWCNTs [31].

\section{Hydrophilicity of the blend membrane}

Water contact angle (CA) is used to characterize the wetting property of top solid surface. The curves of contact angle decay with drop age for the membranes are shown in Figure 4. It can be found that the initial contact angle of the pure CDA membrane top surface (M- 0 )

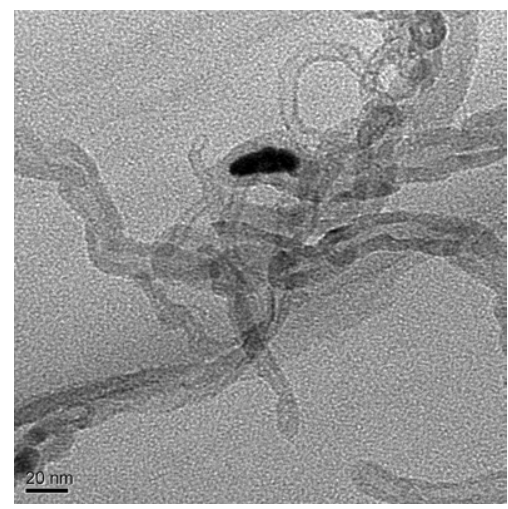

Figure 2: TEM images of MWCNTs.

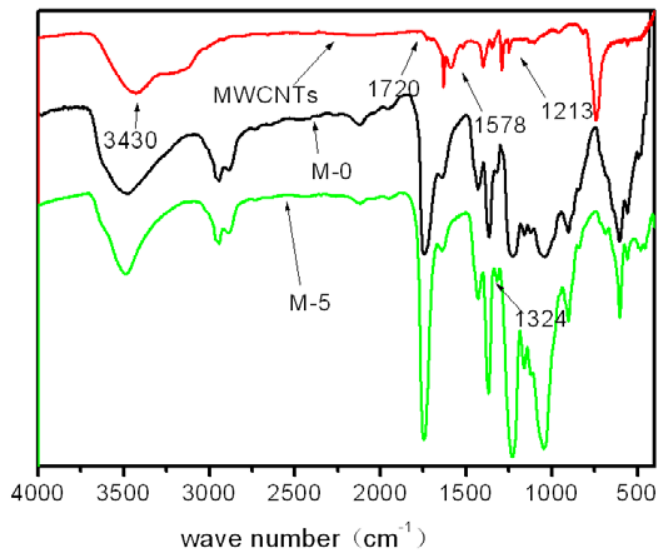

Figure 3: FT-IR spectra of the functionalized MWCNTs, M-0 and M-5.

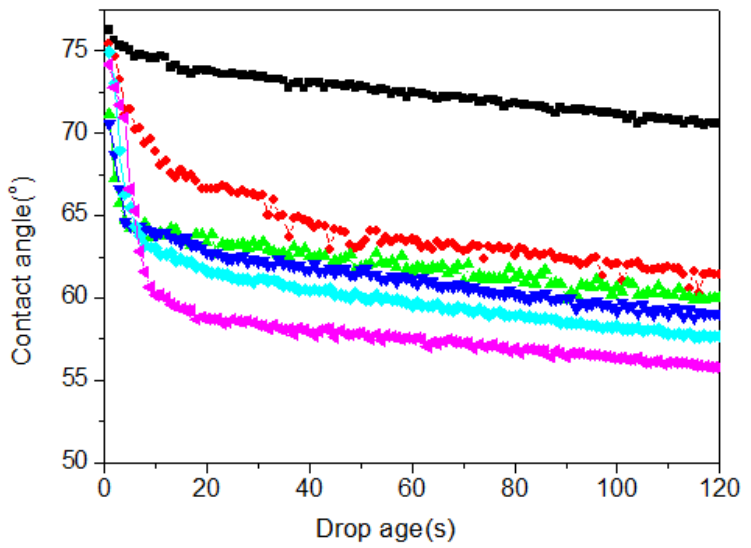

Figure 4: The curves of water contact angle decaying with drop age for the pure CAD (M-0) and the blend membranes, From top to bottom, M-0, M-1, $M-2, M-3, M-4, M-5$ in the order.
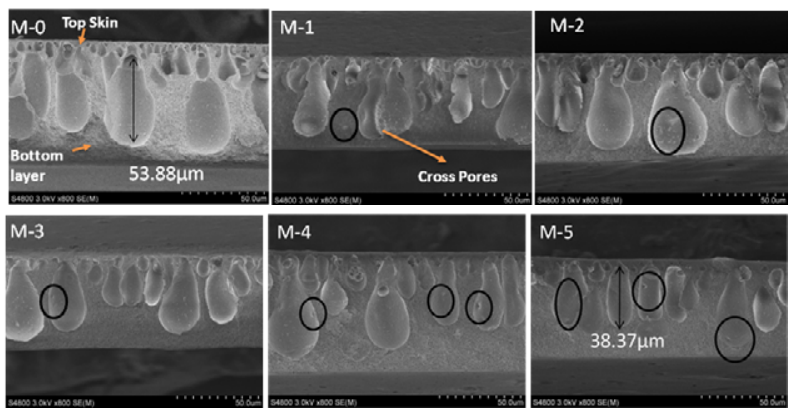

Figure 5: The cross-section SEM images of the pure CAD (M-0) and the blend membranes (M-1, M-2, M-3, M-4 and M-5).

is about $76^{\circ}$ and it decreases only by $6^{\circ}$ in 120 s. For the MWCNTs/ CDA hybrid membranes, the initial contact angles of their top surfaces were about the same but decrease quicker in the first 10 s and then flat out. For example, the initial contact angle of M-4 top surface was about $75^{\circ}$, which drop $12^{\circ} \mathrm{C}$ in the first $10 \mathrm{~s}$ and then slowly decreases $57^{\circ} \mathrm{C}$ in 120 s, indicating improved hydrophilicity from the pure CDA membrane. Similar phenomenon had been reported by Choi et al. [32]. This result can be explained by the fact that during the phase inversion process, hydrophilic MWCNTs migrated spontaneously to the membrane/water interface to reduce the interface energy [33].

\section{Morphology of the hybrid membrane}

In order to understand the influence of MWCNTs on the final membrane structure, the cross-section of the prepared MWCNTs/ CDA blend membranes were observed using scanning electron microscopy (SEM). The cross-section images of membranes are shown in Figure 5. As shown in Figure 5, there are three distinguished sections in the typical cross-sections of the membranes, the thin top skin layer, the porous middle section and the dense bottom section. The thin top skin layer was first formed when exposed to air and then further solidified by immersed into non-solvent. The porous middle section containing finger-like pores was formed by delayed phase separation caused by mutual diffusion of water and organic solvents in the phase inversion process. The dense bottom sponge skin sections were formed 
Citation: Zhao X, Sheng J, Zhu L, Zhang A, Xue L (2015) Influence of Functionalized Multiwall Carbon Nano-Tube on the Structure and Performance of Cellulose Di-Acetate Based Forward Osmotic Membrane for Desalination Process. J Membra Sci Technol 5: 135. doi:10.4172/21559589.1000135

Page 4 of 6

by extraction of solvent from solution and precipitation of polymer skins by water.

The dense top sponge skin sections were formed by immediate extraction of solvent from solution and precipitation of polymer skins by water. Obviously, faster diffusion tends to form thinner top skin sections with smaller surface pores which means the faster the diffusion is, the thinner the top skin is. The concentration of MWCNTs ranged in $1,2,3,4$ and $5 \mathrm{wt} \%$ relative to the weight of CDA, the thickness of the top dense skin section decreased from $3.33 \mu \mathrm{m}(\mathrm{M}-0)$ to $1.66 \mu \mathrm{m}$ (M-1) first, then rising from $3.35 \mu \mathrm{m}$ (M-2) to $6.65 \mu \mathrm{m}$ (M-5). At the same time, by increasing amount of MWCNTs in CDA matrix, the lengths of finger-like pores decreased from $53.88 \mu \mathrm{m}$ to $38.37 \mu \mathrm{m}$ and the thickness of the dense bottom sections was increased.

The distribution of MWCNTs in the membranes could also be observed as indicated by the blank circles in Figure 5. However it is not clearly, the surface morphology of the formed membranes were observed further by Microscope photographs. When the concentration of MWCNTs in the casting solution was $1 \mathrm{wt} \%$, the distribution of MWCNTs on the surface of the MWCNTs/CDA blend membrane was relatively more uniform. When the top surface photographs of prepared membranes were compared as presented in Figure 6. By increasing amount of mixed MWCNTs in CDA matrix, the membrane top surface became darker. Additionally, the dark particles of agglomerated MWCNTs were more visible with the increasing the
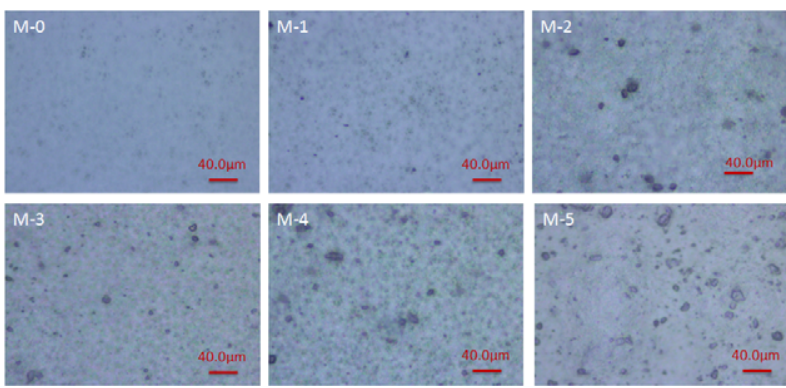

Figure 6: Microscope photographs of surface of membranes with different blend compositions. content of MWCNTs.

AFM was used for morphological characterization of the membrane surfaces. Both the three-dimensional and plane AFM images are shown in Figure 7. According to the AFM images, the top surface of membrane was rather rough. It also shows that the mean square roughness of the membranes firstly increases and then drops as MWCNTs are increased. For M-0, M-1, M-2, M-3, M-4 and M-5 membranes, the mean square roughness is $28.2 \mathrm{~nm}, 52.9 \mathrm{~nm}, 49.1 \mathrm{~nm}, 46.3 \mathrm{~nm}, 36.0 \mathrm{~nm}$, and 23.5 $\mathrm{nm}$, respectively. When the concentration of MWCNTs in the casting solution is $1 \mathrm{wt} \%$, the surface of the MWCNTs/CDA blend membrane has the highest surface roughness. When the content of MWCNTs is increased further, especially past $1 \mathrm{wt} \%$, the top skin surface starts to become smooth again. This is probably due to the contrary effects caused by increased hydrophilicity and increased viscosity by the added hydrophilic MWCNTs. First, the effect of increased hydrophilicity with increased modified MWCNTs can increase the exchange rates of water diffusion during phase-inversion. This makes MWCNTs evenly concentrated on the top surface of CDA membrane to form nodular structure and becomes rougher [34,35]. After MWCNTs content past $1 \mathrm{wt} \%$, the viscosity of the blend solutions increased along with the content of MWCNTs, which usually retards the exchange of solvent and non-solvent, making the membrane surface effectively smoother [36].

\section{Impact of MWCNTs/CDA Ratio on FO Membrane Performance}

The water flux across the employed membrane is one of important factors that affect the efficiency of FO processes. It is closely related to the number and size of the FO unit and even the energy required to pump water through the unit. In our experiments, to evaluate osmosis performance of membranes with MWCNTs, an aqueous solution of $\mathrm{NaCl}$ at an initial concentration of $2.0 \mathrm{M}$ was used as the draw solutions, whereas the feed solution was de-ionized water. The influence of MWCNTs/CDA ratio on the water flux and the reverse solute flux in the FO process were investigated.

The membrane performances in terms of the water flux and the reverse solute flux of $\mathrm{NaCl}$ are presented in Figure 8. The water flux first increased and then decreased with increasing MWCNTs concentration
$M-0: R a=28.2 \pm 2.7 \mathrm{~nm}$

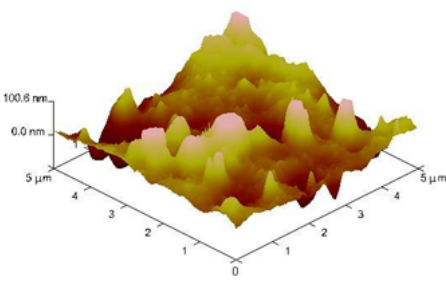

$M-3: R a=46.3 \pm 5.9 \mathrm{~nm}$

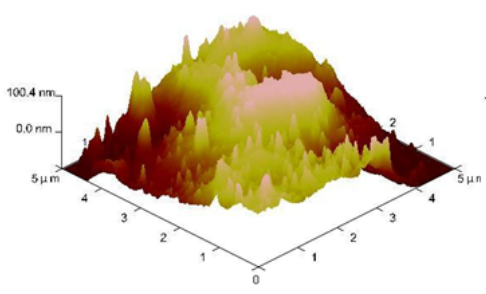

$M-1: R a=52.9 \pm 5.1 \mathrm{~nm}$

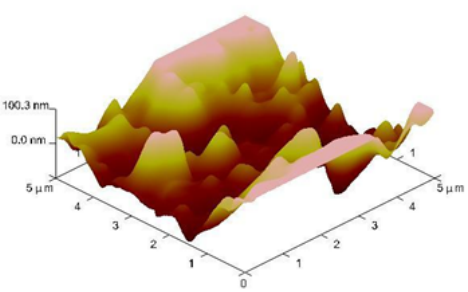

$M-4: R a=36.0 \pm 4.5 \mathrm{~nm}$

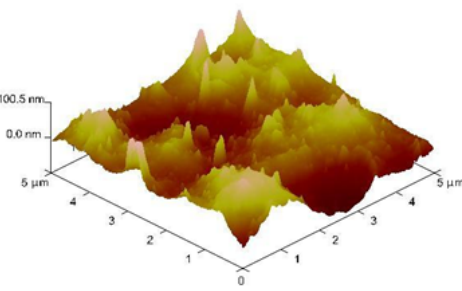

$M-2: R a=49.1 \pm 6.4 \mathrm{~nm}$

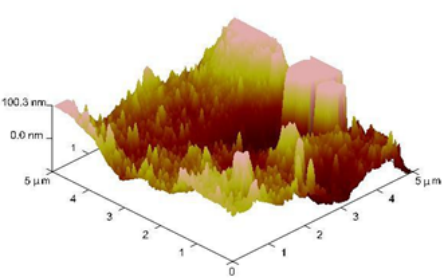

$M-5: R a=23.5 \pm 6.6 \mathrm{~nm}$

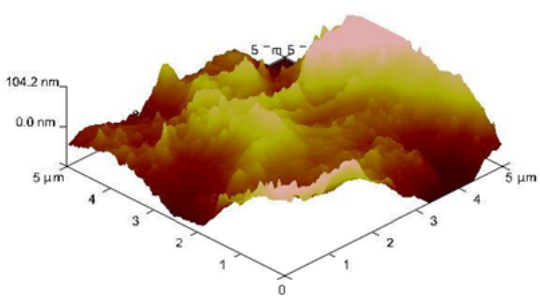

Figure 7: The surface AFM 3D images of different FO membranes from M-0 to M-5 and their mean surface roughness. 
Citation: Zhao X, Sheng J, Zhu L, Zhang A, Xue L (2015) Influence of Functionalized Multiwall Carbon Nano-Tube on the Structure and Performance of Cellulose Di-Acetate Based Forward Osmotic Membrane for Desalination Process. J Membra Sci Technol 5: 135. doi:10.4172/21559589.1000135

Page 5 of 6
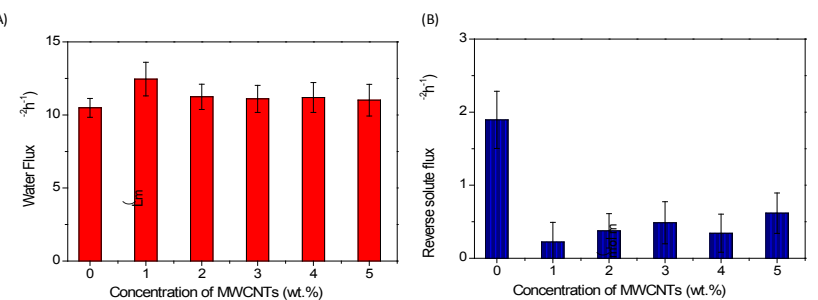

Figure 8: (A) The water flux of different FO membranes with varied MWCNTs/ CDA ratios in the membrane; $(B)$ the reverse solute flux of different membranes with varied MWCNTs/CDA ratios in the membrane, using $2 \mathrm{M} \mathrm{NaCl}$ solution as draw solution and de-ionized water as feed solution.

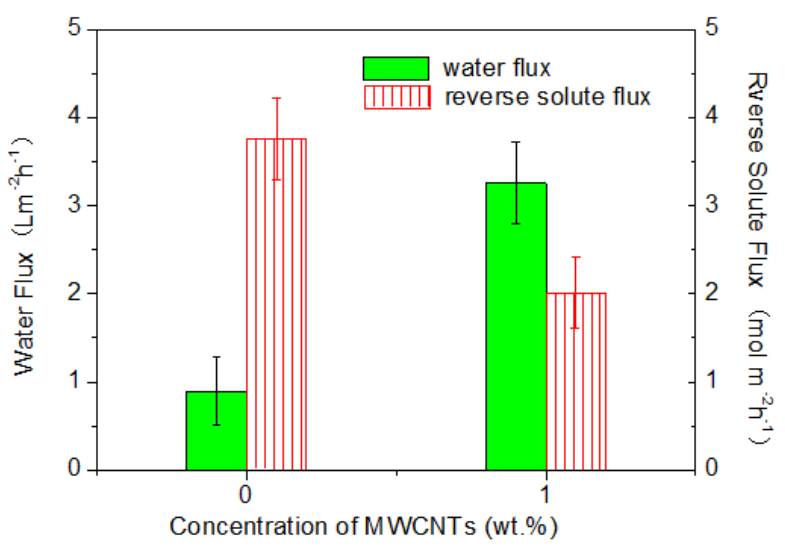

Figure 9: The water flux and reverse solute flux of $\mathrm{M}-1$ using $2 \mathrm{M} \mathrm{NaCl}$ solution as draw solution, $3.5 \mathrm{wt} \%$ simulated seawater solution as feed solution.

in Figure 8A. The water flux of M-1 was higher than that of other membranes because it had higher surface roughness, more uniform distribution of MWCNTs and more cross pores, as discussed in the previous section.

Figure $8 \mathrm{~B}$ shows the reverse solute fluxes of the membranes. The entire blend membranes achieved lower reverse solute flux than that of $\mathrm{M}-0$, indicating the active top skin layers formed in the blend membranes was denser without leakage, the same as shown in SEM, Optical Microscopy, and AFM. Compared with pure CDA FO membrane, the blended membrane of M-1 shows both higher water flux and lowest reverse solute flux, leading to higher potential for applications in lower cost FO water treatment systems.

\section{Osmotic seawater desalination}

In order to test the feasibility of using these high performance FO membranes in sea water desalination processes, $3.5 \mathrm{wt} \%$ simulated seawater solution was used as feed solution, $2 \mathrm{M} \mathrm{NaCl}$ solution was used as draw solution. The osmotic water flux and reverse solute flux of M-0 and M-1 in the FO process were investigated. As shown in Figure 9, the water flux $\left(J_{w}\right)$ of M-0 and M-1 was 0.89 and $3.26 \mathrm{~L} / \mathrm{m}^{2} \cdot \mathrm{h}$ under FO mode, respectively. And the reverse solute flux $\left(\mathrm{J}_{\mathrm{s}}\right)$ of M-0 and M-1 was 3.75 and $2.01 \mathrm{~mol} / \mathrm{m}^{2} \mathrm{~h}$, respectively. It is interesting find that the water flux of M-1 was $366 \%$ higher than that of M-0, and the reverse solute flux of M-1 was $53 \%$ lower than that of M- 0 . The results demonstrated that the addition of MWCNTs into the CDA substrate can lead to better performance of the FO membrane. Compared with pure CDA membrane, M-1 has higher water flux and lower reverse solute flux, which may be attributed to the combined effects from the existence of longer central porous section, the relatively more uniform distribution of MWCNTs, rougher top surfaces and denser top skin layer with more hydrophilic MWCNTs nano-channels.

\section{Conclusions}

The MWCNTs/CDA blend membranes were prepared by the classical phase-inversion method and the performances of the obtained membranes were investigated. It was found that the content of functionalized MWCNTs was an important factor which influenced the hydrophilicity of the blend membranes. SEM, Optical Microscopy, and AFM images of the blend membranes showed that the cross-section morphology changed with the content of functionalized MWCNTs. In the presence of functionalized MWCNTs, the hydrophilicity and the reverse solute flux of the FO membranes could be greatly improved without significantly affecting the pure water flux. With the addition of only about $1 \mathrm{wt} \%$ MWCNTs, the water flux of membrane-1 was increased from $10.5(\mathrm{M}-0)$ to $12.5 \mathrm{~L} / \mathrm{m}^{2} \mathrm{~h}$, while its reverse solute flux was reduced from $1.8(\mathrm{M}-0)$ to below $0.3 \mathrm{~mol} / \mathrm{m}^{2} \mathrm{~h}$. Compared with pure CDA membrane, the membranes with $1 \mathrm{wt} \%$ MWCNTs(M-1) showed $366 \%$ higher water flux and 53\% lower reverse solute flux, which may be attributed to the combined effects from the existence of longer central porous section, rougher top surfaces and denser top skin layer with more hydrophilic MWCNTs nano-channels. These results suggest that CDA bases FO membranes modified with functionalized MWCNTs could possess good potential to be further developed for practical applications in the sea water desalination processes.

\section{Acknowledgment}

The authors appreciate financial supports from National Natural Science Foundation of China (No. 2014GA701029), Bureau of Science and Technology of Ningbo (No. 2014B70023 and 2013C910012) and Ningbo Natural Science Foundation (2014A610141, 2014A610138, 2013B320011 and 2012B71048).

\section{References}

1. Mi B, Elimelech $M(2008)$ Chemical and physical aspects of organic fouling of forward osmosis membranes. J Membr Sci 320: 292-302.

2. Lay WCL, Chong TH, Tang CY, Fane AG, Zhang J, et al. (2010) Fouling Propensity of Forward Osmosis: Investigation of the Slower Flux Decline Phenomenon. Water Sci Technol 61: 927-936

3. Cornelissen ER, Harmsen D, De Korte KF, Ruiken CJ, Qin JJ, et al. (2008) Membrane fouling and process performance of forward osmosis membranes on activated sludge. J Membr Sci 319: 158-168.

4. Mi B, Elimelech M (2010) Organic fouling of forward osmosis membranes: Fouling reversibility and cleaning without chemical reagents. J Membr Sci 348: 337-345.

5. McCutcheon JR, McGinnis RL, Elimelech M (2005) A novel ammonia-carbon dioxide forward (direct) osmosis desalination process. Desalination 174: 1-11.

6. Kessler JO, Moody CD (1976) Drinking Water from Sea Water by Forward Osmosis. Desalination 18: 297-306.

7. Beaudry EG, Lampi KA (1990) Membrane technology for direct osmosis concentration of fruit juices. Food Technol 44: 121

8. Dova MI, Petrotos KB, Lazarides HN (2007) On the direct osmotic concentration of liquid foods. Part II. Development of a generalized model. J Food Eng 78: 431-437.

9. Dova MI, Petrotos KB, Lazarides HN (2007) On the direct osmotic concentration of liquid foods. Part I. Impact of process parameters on process performance. J Food Eng 78: 422-430

10. Anderson DK (1977) Concentration of dilute industrial waste by direct osmosis Master of Science, University of Rhode Island, Providence.

11. Votta F, Barnett SM, Anderson DK (1974) Concentration of Industrial Waste by Direct Osmosis: Completion Report, Providence. 
Citation: Zhao X, Sheng J, Zhu L, Zhang A, Xue L (2015) Influence of Functionalized Multiwall Carbon Nano-Tube on the Structure and Performance of Cellulose Di-Acetate Based Forward Osmotic Membrane for Desalination Process. J Membra Sci Technol 5: 135. doi:10.4172/21559589.1000135

12. Holloway RW, Cath TY, Dennett KE, Childress AE (2005) Forward osmosis for concentration of anaerobic digester centrate. Proceedings of the AWWA Membrane Technology Conference and Exposition, Phoenix, Arizona, USA.

13. Lee KL, Baker RW (1981) Membranes for power generation by pressureretarded osmosis. J Membr Sci 8: 141-171.

14. Jellinek HHG, Masuda H (1981) Osmo-power. Theory and performance of an osmo-power pilot plant. Ocean Eng 8: 103-128.

15. Loeb S (2002) Large-scale power production by pressure-retarded osmosis, using river water and sea water passing through spiral modules. Desalination 143: $115-122$.

16. Theeuwes F, Yum SI (1976) Principles of the design and operation of generic osmotic pumps for the delivery of semisolid or liquid drug formulations. Ann Biomed Eng 4: 343-353.

17. Wright JC, Johnson RM, Yum SI (2003) DUROS ${ }^{\circledR}$ osmotic pharmaceutical systems for parenteral and site-directed therapy. Drug Deliv Technol. Drug Delivery Technol 3: 64-73.

18. Razmjou A, Simon GP, Wang H (2013) Effect of particle size on the performance of forward osmosis desalination by stimuli-responsive polymer hydrogels as a draw agent. Chem Eng J 215: 913-920.

19. Yen SK, MehnasHaja F (2010) Study of draw solutes using 2-methylimidazolebased compounds in forward osmosis. J Membr Sci 364: 242-252.

20. Qiu S, Wu L, Pan X, Zhang L, Chen H, et al. (2009) Preparation and properties of functionalized carbon nanotube/PSF blend ultrafiltration membranes. J Membr Sci 342 165-172

21. Shirazi Y, Tofighy MA, Mohammadi T (2011) Synthesis and characterization of carbon nanotubes/poly vinyl alcohol nanocomposite membranes for dehydration of isopropanol. J Membr Sci 378: 551-561.

22. Mauter MS, Elimelech M (2008) Environmental Applications of Carbon-Based Nanomaterials. Environ Sci Technol 42: 5843-5859.

23. Wang X, Chen X, Yoon K, Fang D, Hsiao BS, et al. (2005) High Flux Filtration Medium Based on Nanofibrous Substrate with Hydrophilic Nanocomposite Coating. Environ Sci Technol 39: 7684-7691.

24. Moniruzzaman M, Winey KI (2006) Polymer Nanocomposites Containing Carbon Nanotubes. Macromolecules 395: 194-5205.
25. Shawky HA, Chae, Lin S, Wiesner MR (2011) Synthesis and characterization of a carbon nanotube/polymer nanocomposite membrane for water treatment Desalination 272: 46-50.

26. Sun Y, Xue L, Zhang Y, Zhao X, Huang Y, et al. (2014) High flux polyamide thin film composite forward osmosis membranes prepared from porous substrates made of polysulfone and polyethersulfone blends. Desalination 336: 72-79.

27. Tiraferri A, Kang Y, Giannelis EP, Elimelech M (2012) Highly Hydrophilic ThinFilm Composite Forward Osmosis Membranes Functionalized with SurfaceTailored Nanoparticles. ACS Appl Mater Interfaces 4: 5044-5053.

28. McCutcheon JR, Elimelech MA (2008) Influence of membrane support layer hydrophobicity on water flux in osmotically driven membrane processes. $J$ Membr Sci 318: 458-466.

29. Zhao W, Li M, Zhang Z, Peng HX (2010) EMI shielding effectiveness of silve nanoparticle-decorated multi-walled carbon nanotube sheets. Int J Smart Nano Mat $1249-260$

30. Jiang X, Gu J, Bai X, Lin L, Zhang Y(2009) The influence of acid treatment on multi-walled carbon nanotubes. Pigm Resin Technol 38: 165-173

31. Yin J, Zhu G, Deng B(2013) Multi-walled carbon nanotubes (MWNTs) polysulfone (PSU) mixed matrix hollow fiber membranes for enhanced water treatment. J Membr Sci 437: 237-248.

32. Choi JH, Jegal J, Kim WN (2007) Modification of Performances of Various Membranes Using MWNTs as a Modifier. Macromol Symp 249-250: 610-617.

33. Sun M, Su Y, Mu C, Jiang Z (2010) Improved Antifouling Property of PES Ultrafiltration Membranes Using Additive of Silica-PVP Nanocomposite. Ind Eng Chem Res 49: 790-796.

34. Han MJ, Nam ST(2002) Thermodynamic and rheological variation in polysulfone solution by PVP and its effect in the preparation of phase inversion membrane. J Membr Sci 202: 55-61.

35. Wienk IM, Boom RM, Beerlage MA, Bulte AMW, Smolders CA, et al. (1996) Recent advances in the formation of phase inversion membranes made from amorphous or semi-crystalline polymers. J Membr Sci 113: 361-371.

36. Choi JH, Jegal J, Kim WN (2006) Fabrication and characterization of multiwalled carbon nanotubes/polymer blend membranes. J Membr Sci 284: 406415. 